\title{
NOTE SUR LE GENRE HADJELIA SEURAT 1916 (NÉMATODES-SPIRURIDAE)
}

\author{
Par Alain G. CHABAUD et Yvonne CAMPANA
}

La diagnose d'espèces décrites par d'anciens auteurs ne repose parfois que sur une erreur d'observation ou sur une anomalie individuelle qu'on est obligé de considérer comme spécifique, puisque Jes types sont généralement perdus. Une telle espèce est souvent décrite de façon correcte par un auteur plus moderne, mais, si le lieu de récolte et la nature de l'hòte sont'différents, elle est fatalement considérée comme une espèce nouvelle. La nomenclature ne peut alors être rétablie que par l'étude de spécimens répondant aux conditions suivantes : $a$ ) hôte et localité géographique correspondant exactement à ceux donnés par l'ancien auteur ; $b$ ) détermination conduisant à l'espèce récemment décrite et ne s'opposant à la description de la première que par des caractères pouvant prêter à erreur.

Notre matériel étant spécialement favorable à cet égard, nous pouvons établir dans ce travail l'identité des espèces Hadjelia truncata (Creplin 1825) et $H$. inermis (Gedoelst 1919). Dans une seconde partie, après avoir revu rapidement la structure céphalique telle qu'elle est décrite dans les différentes espèces du genre, nous cherchcns à définir les caractères génériques d'Hadjelia et nous proposons le rétablissement du genre Excisa Gendre 1928.

Morphologie. - Les spécimens européens ont été récoltés sous la membrane du gésier de Upupa epops L. à Banyuls (PyrénéesOrientales), le 28 avril 1950 (1). Ils ont été comparés aux exemplaires de la collection C. Desportes provenant d'un « petit calao » de Daloa (Côte d'Ivoire), tué le 17 octobre 1947.

Aucun caractère ne permet de différencier les deux lots. Les mensurations effectuées sur chacun de ceux-ci apportent des chiffres

(1) Nous signalons ici la présence en France de l'espèce Sicarius dipterum (Popowa 1927) qui vivait en compagnie de l'Hadjelia dans le gésier de cette Huppe. S. dipterum décrit au Turkestan a déjà été retrouvé en Chine par Li en 1934 .

Annales de Parasitologie, $\mathrm{T}, \mathrm{XXV}, \mathrm{N}^{08} 5-6,1950$. 
qui correspondent parfaitement à ceux donnés par les auteurs précédents pour Hadjelia inermis (Gedoelst 1919, Gendre $1922 b$ ) et pour $H$. truncata (Singh 1949) (1).

L'extrémité céphalique est identique, quelle que soit l'origine du matériel; elle porte deux pseudo-lèvres, deux interlabia courtes, huit papilles et deux amphides. Le bord buccal de chacune des pseudo-lèvres est formé par trois lobes : les deux submédians à

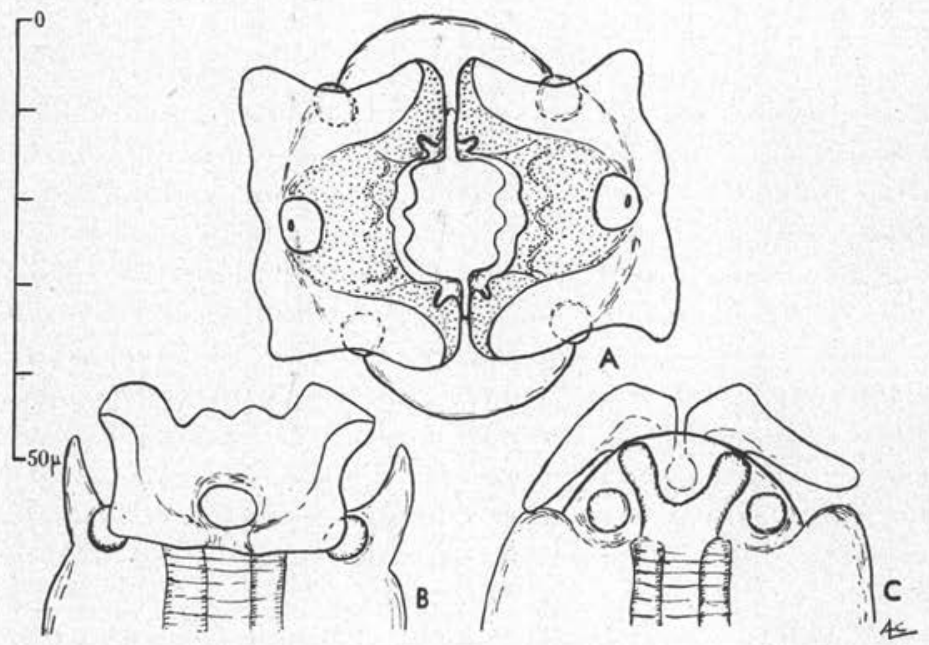

Hadjelia truncate (Creplin 1825) de Upupa epops Banyuls.

Femelle, extrémité céphalique. $A$ : vue apicale. $B$ : vue latérale. $C$ : vue ventrale

bord rectiligne s'opposent étroitement à ceux de la paire correspondante; le lobe latéral, incisé en son milieu, est au contraire largement séparé de son homologue et limite avec celui-ci l'ouverlure buccale. Il existe quatre petites papilles autour de la bouche, chacune étant insérée à l'union du lobe latéral et d'un lobe submédian. Chaque pseudo-lèvre est ornée latéralement d'un plateau rectangulaire transparent, qui est libre sur trois bords et fixé seulement au niveau du pourtour buccal et sur l'amphide. Les quatre grosses papilles submédianes du cycle externe ne sont donc visibles

(1) Nous ne retenons pas le chiffre de $9,5 \mathrm{~mm}$. donné pour la lougueur du vagin dans le mémoire de Singh et qui paraît être une erreur de typographie. L'ovéjecteur de nos spécimens mesure 1,4 à $1,8 \mathrm{~mm}$. pour sa portion impaire et $350 u$ de la bifurcation des trompes à la naissance d'un utérus, ce qui correspond à peu près aux mensurations de Gendre $1922 b$. 
en vue apicale que par transparence à travers le plateau. Les amphides, au contraire, perforent cette membrane et font saillie sur le plan superficiel. La zone des pseudo-lèvres accolée au parenchyme céphalique est beaucoup plus réfringente et forme un triangle dont la base est constituée par le bord de la bouche et le sommet par le plateau amphidien.

Les interlabia très courtes ont un contour régulièrement circulaire. L'échancrure décrite par certains auteurs est due à deux écailles plus réfringentes incluses dans l'épaisseur de chaque interlabia.

En conclusion, la structure céphalique de ces spécimens correspond point pour point aux excellentes descriptions données par Gedoelst (1919) et par Gendre $(1922 a)$ pour $H$. inermis. Nous ne pouvons que les compléter en signalant deux détails accessoires: la présence de quatre petites papilles sur le cycle interne et l'existence d'un plateau rectangulaire transparent annexé aux pseudolèvres.

Mise en synonymie de $\mathrm{H}$. inermis avec $\mathrm{H}$. truncata. - Deux sorles de faits paraissent s'opposer à cette mise en synonymie :

a. - La taille des oufs classiquement de $27 \mu$ chez $H$. truncata. Ni Creplin (1825), ni Mueller (1897) ne donnent de dimensions pour les œufs. Le chiffre de $27 \mu$ admis jusqu'à maintenant repose donc uniquement sur l'observation de Dujardin (1845). Il semble que cet auteur ait eu affaire à des oufs non matures ou de taille anormalement petite, car les nòtres atteignent $57 \mu$. Nous avons déjà pu fournir un exemple du même ordre en signalant pour Seuratia shipleyi (Stossich 1900) des wufs de $45 \times 22 \mu$, alors que la description de Seurat indique $25 \times 16 \mu$ (Desportes, Chabaud et Campana, 1949).

b. - Les redescriptions récentes de Singh admettant deux espèces distinctes.

Singh (1949) a redécrit $H$. truncata provenant de Coracias benghalensis et de Upupa epops, et $H$. inermis provenant de Halcyon smyrnensis et considère les deux espèces comme distinctes.

La femelle de $H$. inermis étant manifestement immature, les seules différences qu'il est possible de relever entre les deux descriptions dans le texte de cet auteur sont les suivantes : "cadre buccal » à huit lobes chez $H$. truncata et à six lobes chez H. inermis ; paire de petites papilles supplémentaires près de la pointe caudale que l'auteur signale chez $H$. inermis et qu'il ne mentionne pas chez H. truncata. 
Ces caractères ne nous paraissent pas avoir une valeur spécifique : la paire de papilles supplémentaires (Phasmides ?) est souvent difficile à voir; dans un mème lot, elle semble manquer chez certains spécimens et apparait relativement bien chez d'autres. En outre, Gendre $(1922 a)$ a bien montré que le "cadre buccal 》 de Seurat n'a pas de valeur et ne se forme que si la tête est rétractée. Nous ne pouvons donc pas suivre les conclusions de Singh et croyons pouvoir mettre $H$. inermis en synonymie de $H$. truncata.

Structure céphalique des différentes espèces d'Hadjelia. - II reste sept espèces classées dans le genre Hadjelia.

H. lhuillieri Seurat 1916, espèce type, est probablement identique à H. truncata, ainsi que Cram (1927) l'a déjà suggéré. La structure céphalique a été décrite et figurée de façon un peu schématique, mais son étude ne semble pas devoir apporter de caractères distinctifs avec cette dernière.

H. parva Gendre $1922 b$ est encore du même groupe, mais semble se distinguer par un corps et surtout une extrémité céphalique plus trapus.

H. excisa (Molin 1860), redécrit par Seurat (1914) et Yamaguti (1941), est au contraire extrêmement différent. Il existe huit papilles sur le cycle externe; les interlabia sont profondément échancrées et les pseudo-lèvres portent chacune trois grosses dents qui s'engrènent avec celles du côté opposé. En outre, la vulve située au milieu du corps et l'anatomie de l'appareil génital mâle le rapprochent des Habronema et l'éloignent des autres Hadjelia.

H. coragypis Sandground 1929 se rapproche de H. excisa par la place de la vulve et même par certains caractères de la bourse caudale du mâle, telles que les petites arêtes cuticulaires qui ornent sa surface. L'extrémité céphalique est malheureusement décrite de façon très succincte : "Mouth typically limited by two lateral trilobed lips », et l'on ne peut pas savoir si elle est du type $H$. truncata ou du type $H$. excisa. Il en est de même pour deux espèces décrites plus récemment: $H$. annulata Harwood 1933, où la bouche est limitée par deux grandes lèvres bilobées, et $H$. curvata Vaz et Pereira 1935, avec deux lèvres latérales trilobées sans papilles.

La structure buccale des Hadjelia peut donc revêtir au moins deux aspects très différents : le type truncata (avec les espèces truncata, lhuillieri et parva) et le type excisa, cette structure restant à peu près inconnue pour les trois autres espèces. Le genre proche Parhadjelia, créé par Lent et Freitas (1939), présente des caractères intermédiaires, mais se rapproche plus du type truncata. 
Définition du genre Hadjelia. - La définition générique d'Hadjelia devient done maintenant très difficile à préciser. On ne peut conserver celle proposée par Seurat (1916) en ce qui concerne l'extrémité céphalique, puisque le « cadre buccal 》 est très probablement (cf. Gendre, $1922 a$ ), une formation hétérogène comprenant les amphides et les interlabia reliées par des plis visibles sur certains spécimens contractés. La définition de Chitwood et Wehr (1934) ne peut être retenue, puisqu'elle est basée sur le caractère " interlabia deeply notehed ».

Nous pensons done qu'il faut scinder ce genre hétérogène et qu'il y a avantage à rétablir le genre Excisa Gendre 1928 qui avait été mis en synonymie par Chitwood et Wehr en 1934. Il suffit, dans la définition générique d'Hadjelia donnée par Seurat, d'amender la partie qui concerne l'extrémité céphalique :

Habronematinæ. Pseudo-lèvres très grandes ornées d'appendices cuticulaires transparents. Interlabia courtes non échancrées. Cycle externe formé par quatre papilles submédianes insérées au niveau de la naissance des interlabia.

\section{RÉSUMÉ}

Etude morphologique de l'extrémité céphalique d'Hadjelia truncata (Creplin 1825). Mise en synonymie de l'espèce $H$. inermis (Gedoelst 1919) avec la précédente. La définition du genre Hadjelia est modifiée en ce qui concerne l'extrémité céphalique et nous proposons le rétablissement du genre Excisa Gendre 1928.

\section{BiBLIOGRAPHIE}

ChITwood (B. G.) et WhHR (E. E.). - The value of cephalie structures as characters in nematode classification, with special reference to the superfamily Spiruroiciea. Zeitsch. für Parasit., VII, 1934, 273-335 + 1 pl.

Cвам (E. B.). - Bird parasites of the Nematode suborders Strongylata, Ascaridata and Spirnrata. Buli. U.S. Nai. Mus., 1927, $\mathrm{n}^{\circ} 140$, xvir +465 p.

Creplin (H.). - Observationes de entozois, 1825, x +86 p. +1 pl., Gryphiswaldix.

Desportes (C.), Chabaud (A.-G.) et Campaxa (Y.). - Sur les gongylonèmes de Muride et leurs formes larvaires. Ann. Parasit., XXIV, 1949, 447-459.

DUjardin (F.). - Histoire naturelle des helminthes ou vers intestinaux, 1845, $\mathrm{xv}_{\mathrm{I}}+654 \mathrm{p} .+12$ pl., Paris.

(iedoelst (L.). - Un genre nouveau de Spiruride. C.R. Soc. Biol., LXXXII, 1919, 1145-1148. 
Gendne (E.). - Sur l'identité des genres Iadjelia Seurat et Gilsonia Gedoelst et leurs affinités avec le genre Histiocephalus Diesing. P.V. Soc. Linn. Bordeaux, LXXIII, 1922a, 138-142.

- Sur deux espèces d'Hadjelia. P.V. Soc. Linn. Bordeanx, LXXIV, 1922b, 26-32.

In Joyeux (Ch.), Gendre (E.) et Baer (J.-G.). - Recherche sur les helminthes de l'Afrique Occidentale française. Coll. Soc. Path. Exot., Monogr. II, 1928, Masson.

Harwoon (P. D.). - Some spiruroid nematodes from Texas birds. Trans. Amer. Micr. Soc., LII, 1933, 173-176.

LeNt (H.) et Freitas (J.-F. DE). - Novo nematodeo parasito do pato domestico (Spiruroidea). Bol. Biol. (N.S.), IV, 1939, 178-180.

LI (H. C.). - Report on a collection of parasitic nematodes mainly from North China. Part. II, Spirurcidea. Trans. Amer. Micr. Soc, LIII, 1934, 174-195.

MUeleer (M.). - Helminthologische Mittheilungen. Archiv. für Naturg., I, 1897, $1-26+3 \mathrm{pl}$.

Popowa (K. A.). - Ein neuer Nematode des Wiedehopfes (UFupa epops L.), Habronema dipterum n. sp. (en russe, résumé en allemand). Sammlung Helminth. Arbeiten K. I. Skrjabin gewidmet Moscow, 1927, 180-185.

Sandground (J. H.). - Some new parasitic Nematodes from Yucatan (Mexico), including a new genus of Strongyle from cattle. Bull. Mus, Compar. Zool. Harvard College, LXIX, 1929, 515-524 + 2 pl.

Sivnat (L.-G.). - Sur un nouveau parasite de la eigogne blanche. Bull. Soc. Hist. Nat. Afrique Nord, V, 1914, 75-80.

- Sur un nouveau type de Spiruride. C.R. Sor. Biol., LXXIX, 1916, 517-519.

Singh (S. N.). - Studies on the helminth parasites of birds in Hyderabad state. Nematoda, III. J. Helm., XXIII, 1949, 25-38.

VAZ. (Z.) et Pereili (C.). - Some new brazilian Nematodes. Trans. Amer. Micr. Soc., LIV, 1935, 36-40.

Yamaguti (S.). - Studies on the Helminth Fauna of Japan. Part. 36. Avian Nematodes, II. Japan. J. Zcol., IX, 1941, 441-480.

Institut de Parasitologie de la Faculté de Médecine de Paris.

(Directeur : Professeur H. Galliard).

el Laboratoire Arago, Banyuls-sur-Mer (Directeur : Professeur G. Petit). 